\title{
A HÖLDER TYPE INEQUALITY FOR SYMMETRIC MATRICES WITH NONNEGATIVE ENTRIES
}

\section{G. R. BLAKLEY AND PRABIR ROY}

The element $w=\left(w_{1}, w_{2}, \cdots, w_{n}\right)$ of the $n$-dimensional real euclidean vector space $R_{n}$ is nonnegative if $0 \leqq w_{j}$ for each $j$. If $1 \leqq k \leqq n$ then $w(k)=\left(w(k)_{1}, w(k)_{2}, \cdots, w(k)_{n-1}\right) \in R_{n-1}$ is defined by setting $w(k)_{i}=w_{i}$ if $1 \leqq i<k, w(k)_{i}=w_{i+1}$ if $k \leqq i<n$. The real $n$ by $n$ matrix $S=\left(s_{i j}\right)$ is nonnegative if $0 \leqq s_{i j}$ for each $i, j$. If $1 \leqq k \leqq n$ let $S(k)$ be the $n-1$ by $n-1$ matrix obtained by deleting the $k$ th row and $k$ th column of $S$. $W_{n}$ is the boundary of the nonnegative cone in $R_{n}$ and $U_{n}=\left\{u \in R_{n}:(u, u)=1\right\}$ is the unit sphere.

TheOREM. If $S$ is a nonnegative symmetric $n$ by $n$ matrix, $u \in U_{n}$ is nonnegative and $k$ is a positive integer then $(u, S u)^{k} \leqq\left(u, S^{k} u\right)$. If $k>1$ equality holds if and only if $\boldsymbol{u}$ is a characteristic vector of $\mathbf{S}$ or $\left(\boldsymbol{u}, \boldsymbol{S}^{k} \mathbf{u}\right)$ $=0$.

Proof. There is no loss of generality in ignoring trivial cases and assuming that $k>1, n>1$, that $|\lambda| \leqq 1$ for each characteristic value $\lambda$ of $S$ and that there is a characteristic value $\lambda^{*}$ of $S$ for which $\left|\lambda^{*}\right|=1$. There is thus a nonnegative characteristic $n$-vector $v \in U_{n}$ of $S$ whose corresponding characteristic value $\lambda$ is $1[1, p .80]$. Now proceed by induction on $n$.

If $w \in W_{n} \cap U_{n}$ there is some $j$ such that $w(j) \in U_{n-1}$. If

$$
(w(j), S(j) w(j))^{k}<\left(w(j), S(j)^{k} w(j)\right)
$$

then

$$
(w, S w)^{k}=(w(j), S(j) w(j))^{k}<\left(w(j), S(j)^{k} w(j)\right) \leqq\left(w, S^{k} w\right) .
$$

If, on the other hand, $0<(w(j), S(j) w(j))^{k}=\left(w(j), S(j)^{k} w(j)\right)$ then $w(j)$ is, as a consequence of the induction hypothesis, a characteristic $(n-1)$-vector of $S(j)$ and there is some $\lambda>0$ such that $S(j) w(j)$ $=\lambda w(j)$. Hence $S w=\lambda w+p$, where $p$ is a nonnegative $n$-vector for which $(p, w)=0$. If $w$ is not a characteristic vector of $S$ then $(p, p)>0$ and it is easy to verify, using the symmetry of $S$, that

$$
\left(w, S^{k} w\right) \geqq \lambda^{k}+\lambda^{k-2}(w, S p)=\lambda^{k}+\lambda^{k-2}(p, p)>\lambda^{k}=(w, S w)^{k} .
$$

Thus the truth of the theorem in the $(n-1)$-dimensional case entails its truth for vectors in $W_{n}$.

Received by the editors November 2, 1963 and, in revised form, October 5, 1964. 
Suppose the nonnegative vector $u \in U_{n} \sim W_{n}$ is not a characteristic vector of $S$. Let $m \in U_{n}$ be a nonnegative characteristic vector of $S$ with characteristic value 1 and let $q$ be the unique element of $U_{n}$ orthogonal to $m$ such that $u$ is between $q$ and $m$ in the sense that there is some $\eta_{0}, 0<\eta_{0}<1$, for which $u=\left(1-\eta_{0}^{2}\right)^{1 / 2} m+\eta_{0} q$. Let $\alpha=\left(q, S^{k} q\right)$ $-1, \beta=(q, S q)-1$. Notice that $\beta<0$, for otherwise it would follow from the normalization of $S$ that $q$ would be a characteristic vector of $S$ with characteristic value 1 , whence so would $u$, contrary to assumption. There is some $w \in W_{n} \cap U_{n}$ which lies between $u$ and $\boldsymbol{q}$, that is there is some $\eta_{1}, \eta_{0}<\eta_{1} \leqq 1$, such that $\left(1-\eta_{1}^{2}\right)^{1 / 2} m+\eta_{1} q=w$.

Let $f(\lambda)=\lambda^{k}-\lambda \alpha / \beta-1+\alpha / \beta$ for each real $\lambda$. Then

$$
\begin{aligned}
f(1) & =(m, S m)^{k}-\left(m, S^{k} m\right)=0, \\
f\left(1+\eta_{0}^{2} \beta\right) & =(u, S u)^{k}-\left(u, S^{k} u\right), \text { and } \\
f\left(1+\eta_{1}^{2} \beta\right) & =(w, S w)^{k}-\left(w, S^{k} w\right) \leqq 0
\end{aligned}
$$

as a consequence of the symmetry of $S$. Since $0<1+\eta_{1}^{2} \beta<1+\eta_{0}^{2} \beta<1$ and $f$ is a strictly convex $[2$, p. 75$]$ function of a positive argument strict inequality holds at $u$.

\section{REFERENCES}

1. F. R. Gantmacher, Applications of the theory of matrices, Interscience, New York, 1959.

2. G. H. Hardy, J. E. Littlewood and G. Polya, Inequalities, Cambridge Univ. Press, Cambridge, 1952.

UNIVERSITY OF ILLINOIS AND UNIVERSITY OF WISCONSIN 